\title{
Proposal for a Future Internet Business Model and Regulatory Acts in a Technological Convergence Environment Supporting Continuity of QoS
}

\author{
Ana P. G. Serra ${ }^{1}$, Arthur F. A. Battaglia ${ }^{2}$, Moacyr Martucci Jr. ${ }^{3}$ \\ Polytechnic School University of São Paulo-USP, São Paulo, Brazil \\ Email: apaulacg@uol.com.br, arthur.battaglia@uol.com.br, moacyr.martucci@poli.usp.br
}

Received February 2013

\begin{abstract}
The communications development requires interaction between converging heterogeneous technology environment, with quality and continuity of services to remain competitive. The full implementation of the Future Internet concept implies in the necessity to operate among heterogeneous technology platforms with continuity of QoS (Quality of Service), what leads to the necessity of an innovative business model to support it and new technical mechanisms of vertical handover to ensure the QoS continuity required and expected by final users but, mainly, perceived by them. An innovative business model that requires innovative QoS continuity mechanisms must consider technical and commercial interoperation among many telecommunication services providers, nationally and internationally based. This interaction demands clear rules to be followed by every player along the telecommunication services chain, i.e., it demands a set of regulation acts to guide them and allow their viability.
\end{abstract}

Keywords: Technological Convergence; Business Model; QoS; Regulatory Acts; Continuity of Services; Handover; Future Internet

\section{Introduction}

The sector of communications, for some years, has undergone significant changes requiring interaction between converging heterogeneous technology environment, with quality and continuity of services to remain competitive, because this market requires constantly more technological resources available.

Certainly one of the main propellant engines that boost the constant technological advances is the telecommunication services segment, which develops directed to the services consumers market. This paper considers the consumer market as formed by both kinds of final users: people and enterprises. This is the market that requires, constantly, that more and more telecommunication services will be made available.

The full implementation of the Future Internet concept implies in the necessity to operate among heterogeneous technology platforms with continuity of services and QoS (Quality of Service), what leads to the necessity of an innovative business model to support it and new technical mechanisms of vertical handover (or handoff) to ensure the QoS continuity required and expected by final users but, mainly, perceived by them. A satisfied user with the set of services contracted from a telecommunication ser- vices provider will become loyal to it, ensuring the enterprise market share and propitiating you commercial activity continuity. In this sense, the continuity of QoS perceived by users is a commercial strategic element.

QoS is defined in this paper (regarding to the perception of the end user in an environment of technological convergence) as the quality that should be presented to the user by mobile services and interactive multimedia content provided by service providers in an environment of technological convergence.

The mechanism of vertical handover aims to allow mobility to the users ensuring the continuity of QoS perceived by them along all their displacement. This mechanism needs to be characterized as a global solution, i.e., the mechanism must be generic and technology independent, allowing its adoption in any network environment taking advantage of the capillary already available from legacy networks.

An innovative business model that requires innovative QoS continuity mechanisms must consider, in a situation of free market competition, technical and commercial interoperation among many telecommunication services providers, nationally and internationally based. This interaction demands clear rules to be followed by every player 
along the telecommunication services chain, i.e., it demands a set of regulation acts to guide them and allow their viability.

The objective of this paper is to propose a business model and a set of items to be considered for regulation acts.

\section{Technological Convergence}

In this paper technological convergence is conceptualized of a broadly under the aspect of information systems such as the integrated use of heterogeneous technologies for provision of mobile services and interactive multimedia contents that allows the end user has access to their information, applications, knowledge and services anywhere, over any network access, anytime and using any device [1].

The concept of technological convergence is often confused with the concept of interoperability. The big difference between interoperability and convergence is that interoperability is the ability to work together with other systems and standalone applications, based on accepted standards and used in the market which aims to end systems integration. While convergence is to adjust the same service to different media and heterogeneous technologies, allowing end users to access their information and services anywhere, anytime, any network, any device, through an user interface consistent with adequate quality of service and transparent to the user. This requires fundamentally the ability to mobility, portability of applications and content, interconnection and interoperability between systems, platforms and operators [1].

Technological convergence is composed of factors that are beyond the focus on technology. Technological convergence is composed of three basic factors: 1) technology; 2) business and 3) service [2]. Since the relationship between these three factors is essential because technology is a facilitator to provide services to end users, the company is the entity/provider that produces, distributes and/or management services according to market demand and business rules of the company, and the service is the solution to meet end users. The three factors are considered important, although the first two are more easily reached, if the latter is not available with quality, the first two do not make sense.

Other factors deemed important in the technological convergence, as shown in Figure 1.

The standards establish rules and technology standards are affected by the market, by business and by technological innovation, which drives the creation of rules and well-defined standards for their use, creating the need for regulatory acts for its implementation.

\section{Proposed Business Model}

In Proceedings of the 2010 ITU-T Kaleidoscope Aca-

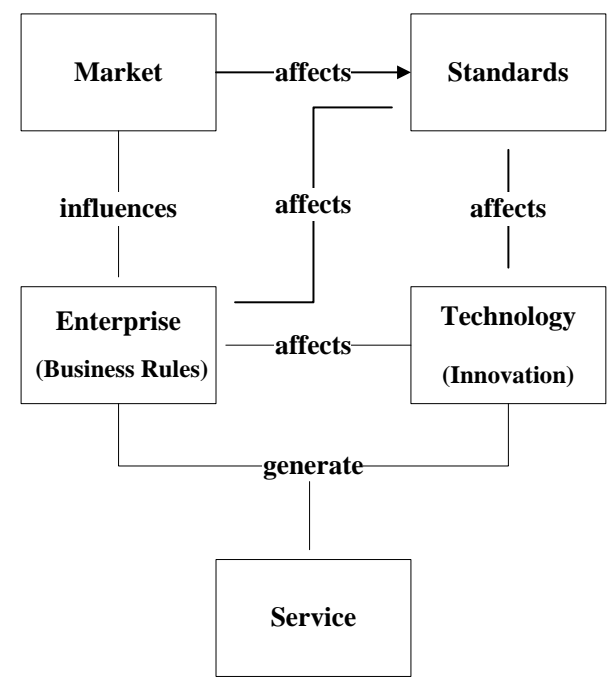

Figure 1. Factors that comprise the technological convergence.

demic Conference, Sakurai [3] proposed a business model based on convergent networks for a user moving within a heterogeneous convergent environment. His proposal is to create a kind of enterprise designated by Service Provider, with the function of monitor the QoS level of the other Providers: Access, Infrastructure and Content. In this model the end user shall relate only to the Service Provider which, in turn, relates to the Access, Infrastructure and Content Providers. The user requests the services desired to the Service Provider, which selects which of other Providers have the best condition to meet the required service with the quality desired by the user at that moment. The operation of Access, Infrastructure and Content Providers becomes transparent to him, that is, becomes imperceptible to the user.

This model establishes the way for a formal relationship among the four kinds of Providers involved. The relationship between the final user and the Service Provider occurs through an USLA (User Service Level Agreement) and between the Service Provider and each one of the other Providers the relationship occurs through a PSLA (Provider Service Level Agreement). This model is shown in Figure 2. Figure 3 shows the logical connections of data flows related to the business model.

The user's mobile device is logically connected to two providers: Access and Service (the physical connection occurs only through the Access Provider).

USLA is a formal agreement between the end user and the Service Provider. Its goal is to define the types of services and levels of QoS that the Service Provider undertakes to provide, in addition to legal provisions.

PSLA is a formal agreement between the Service Provider and each of the Access, Infrastructure and Content Providers establishing technical and commercial parameters, besides the QoS level between them for each service 


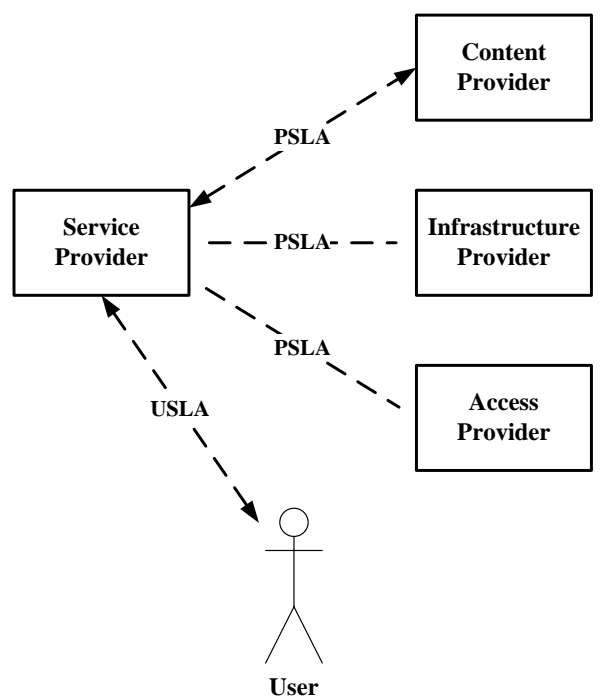

Figure 2. Business model scheme.

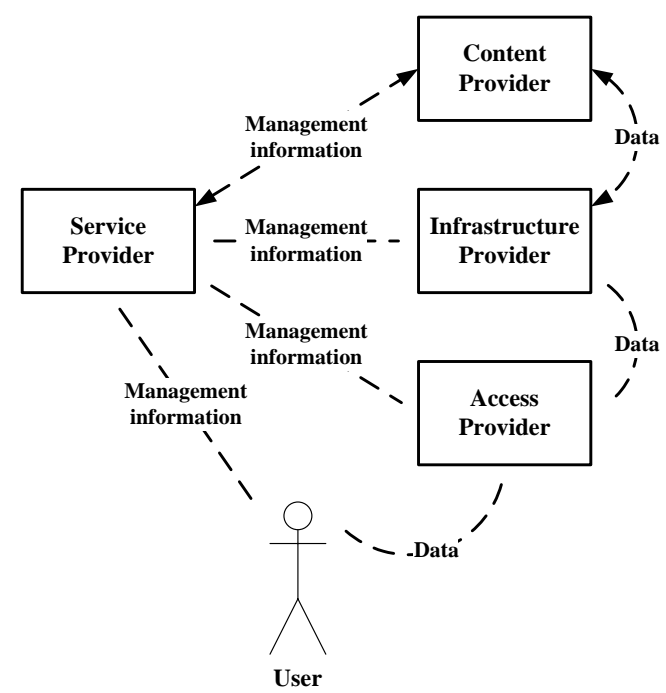

Figure 3. Logical connections among the actors involved.

engaged.

\section{Continuity of QoS through the Vertical Handover Mechanism}

The term handover (or handoff) was intended initially to mobile telephony, designating the mechanism for changing the flow of information between cells during a user displacement without interrupting the connection and seamlessly to him. For an environment of technological convergence is no longer possible to understand the handover only as a mechanism of change in the flow of information only to cell phone, or to a network with homogeneous characteristics. The handover also constitute a mechanism for changing flow of information between heterogeneous networks, or in other words, between different technologies. An example of this can be found in
[4], where is cited the transfer of information between the cellular network and digital TV, allowing users to enjoy mobile TV service.

The handover is divided into two categories: horizontal and vertical.

Horizontal handover occurs when the flow of information is moved between two homogeneous wireless networks, for example, changing the flow of information between Radio Base Stations - RBSs in a cell phone transmission.

Vertical handover occurs when the flow of information is moved between heterogeneous networks. The goal being pursued is that this handover process occurs in order to ensure continuity of service quality to the user seamlessly.

To ensure continuity of service quality is necessary, for Telecommunication Service Providers, maximize the combined use of available resources in heterogeneous networks, such as: WLANs (Wireless Local Area Network), UMTS (Universal Mobile Telecommunications Systems), VANETs (Vehicular ad hoc Network), WiMax (Worldwide Interoperability for Microwave Access) and MANETs (Mobile ad hoc Networks) [5].

The need to trigger a vertical handover mechanism can occur both from the physical displacement of the user and from the need for reconfiguration of links between Providers.

As an example, in the case that the user is positioned in a particular physical location, and accessing a service of his/her choice, the Service Provider selects the best combination of links between Access, Infrastructure and Content Providers to meet user demand.

If the Infrastructure Provider becomes unavailable (for failure by overload hits or for any other reason), for instance, the Service Provider will detect this situation and provide the switching of the flow of information to another Infrastructure Provider which, at that instant, fulfills the conditions necessary to replace the first Infrastructure Provider in all its functions.

Regarding the relationship between the user and the Service Provider, and in a situation of free market competition, a user can hire USLAs with more than one Service Provider to obtain the desired range of services. In this situation, it may occur that the user has hired the same service from two different Service Providers. This situation, in which two different Service Providers are contracted by a single user, can be represented by a universe of two intersecting sets, as illustrated in Figure 4.

When a requested service only belong to the domain administered by Service Provider 1 or, on the other hand, belong only to the domain administered by Service Provider 2, there is no conflict on the decision to which Service Provider fit the mission of triggering the vertical handover. Some parameters involved in the algorithms 


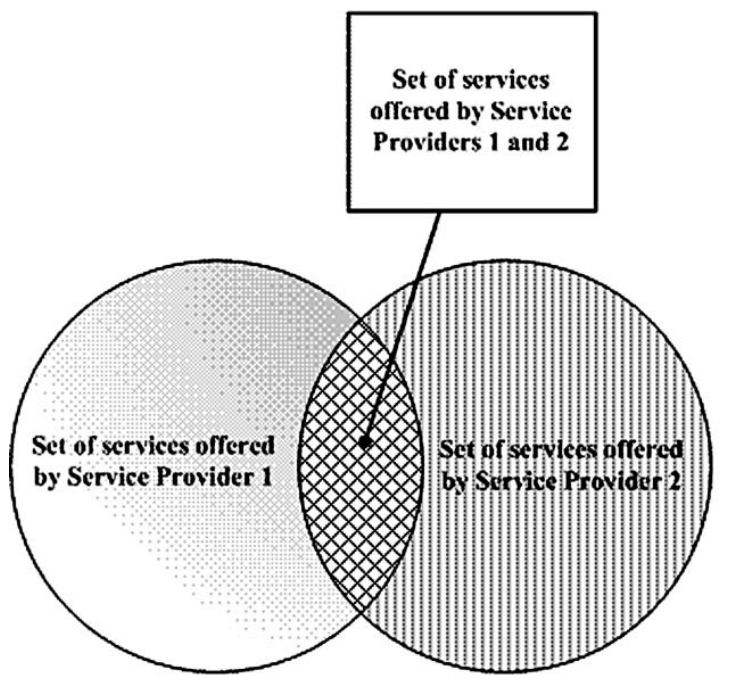

Figure 4. Universe of services offered by Service Providers 1 and 2.

for the traditional decision making processes in handover are: received signal strength, connection time on the network, available bandwidth, power consumption, cost, safety, delay and jitter.

The decision-making process, however, must be enhanced to provide continuity of QoS. Besides the parameters listed in the preceding paragraph, other conditions must be considered, such as those specified in the client USLA and the PSLAs agreed between Providers. In a USLA will be defined, for example, the limits of acceptable service unavailability agreed between the user and the Service Provider for each service contract. This decision process involves algorithms that seek to make the right decision to switch the flow of information at the right time, which implies a set of multiple criteria to be considered.

When a handover is necessary, and its attainment affect the common universe of Providers managed by Service Providers 1 and 2, the decision process should consider one more variable in the decision-making algorithm: the Service Provider which offers the best option for the handover, understanding as best one that offers better technical/commercial conditions for the continuity of service quality and the achievement of stipulated parameters in USLAs, according to user choice policy, based on a prediction algorithm for QoS.

For decision making it is necessary for Service Providers 1 and 2 to exchange information to define the best routing option to execute the handover maintaining continuity and quality of service. Among Service Providers there must be agreements that set the standards for this type of interaction: the Handover Decision Service Providers Agreement - SPHDA [6]. Join a SPHDA implies acceptance of a technical/trade partnership between the two Service Providers involved and hence the access per- mission of a Service Provider to the management information database from the other, and vice versa. A management information database is the repository of all technical occurrences in networks under the administration of the Service Provider. The reasoning developed for two Service Providers can be extended to any number of Service Providers.

\section{Regulatory Acts}

Regulatory acts establish guidelines for application of technical and business procedures aiming the continuity of QoS contracted by the end user. All the documents mentioned before (USLA, PSLA and SPHDA) must have their content regulated. The items that must be considered for an official regulation are shown hereafter.

The USLA is characterized by a set of technical information but, also, by the list of services the user wants to hire:

- specification of the desired services;

- specification of the maximum cost acceptable for each service;

- specification of the level of availability for each hired service;

- specification of the security level for each hired service;

- specification of the response time for each hired service and

- specification of other technical parameters as: bandwidth, loss rate, jitter and transmission velocity.

- The PSLA is characterized by a set of business and technical information that keeps certain resemblance to the SHPDA:

- standardization of the type of management information;

- standardization of the structure and formatting of management information;

- agreement allowing the Service Provider to capture and send management information to the Access, Infrastructure and Content Providers through mobile agents;

- standardization of types of mobile agents that will be used to exchange the management information among providers;

- agreement on the availability of the management information database and

- access to predict the pricing between providers for each service to be executed.

- The SPHDA is characterized by a set of business and technical information that takes part of the decision process to define which of the Service Providers, hired by the same user, will trigger the handover:

- agreement for mutual access to Service Providers management information databases among all signatories of SPHDAs; 
- standardization of identification and authentication codes for access to databases of management information;

- standardization of the type of management information;

- standardization of the structure and formatting of management information;

- standardization of requests for access to a database of management information;

- agreement to management information access through mobile agents;

- standardization of types of mobile agents that will be used to exchange the management information among Providers;

- agreement on the maximum response time for each request for access to a database of management information;

- agreement on the availability of the management information database and access to predict the pricing between providers due to the handover to be executed.

\section{Conclusion}

Open standards such as 3GPP and NGN pave the way for the development of telecommunications services providing a further development of the market for telecommunications services offering services with better quality. Besides the 3GPP and NGN there is a need, however, for the adoption of other additional standards as function of technological changes and behavioral patterns of the us- ers. These standards need regulatory acts for its implementation.

\section{REFERENCES}

[1] A. P. G. Serra, "Method for Identifying Parameters of Quality of Services Applied to Mobile and Interactive Services,” Ph.D. Thesis, Escola Politécnica da Universidade de São Paulo, Brazil, 2007.

[2] S. Shepard, "Telecommunications Convergence: How to Profit from the Convergence of Technologies, Services and Companies,” McGraw-Hill, New York, 2000, 371 p.

[3] C. A. Sakurai, M. Martucci Jr. and A. H. Hirakawa, "Telecommunication Business Model for Converged Networks Focusing Final Users,” Proceedings of 2010 ITU-R Kaleidoscope Academic Conference, pp. 241-248.

[4] R. Corjava, "QoS Analysis in Overlay Bluetooth WiFi Networks with Profile Based Vertical Handover," IEEE Transactions on Mobile Computing. Vol. 5, No. 12, 2006, pp. 1679-1690. http://dx.doi.org/10.1109/TMC.2006.187

[5] S. Lee, K. Sriram, K. Kim, Y. Kim and N. Golmie, "Vertical Handoff Decision Algorithms for Providing Optimized Performance in Heterogeneous Wireless Networks," IEEE Transactions on Vehicular Technology, Vol. 58, No. 2, 2009.

[6] A. F. A. Battaglia, J. M. M. Battaglia, M. Martucci Jr. and A. H. Hirakawa, "Final Users Perceived QoS on Mobile Convergent Multimedia Services,” In: WSEAS, 10th Recent Researches in Communications, Automation, Signal Processing, Nanotechnology, Astronomy \& Nuclear Phisycs," Cambridge, February 2011, pp. 48-53. 